\title{
Complexity of intermediate size fermion/boson gases
}

\author{
G.L. Ferri ${ }^{2}$, F. Pennini ${ }^{1,2}$, A. Plastino ${ }^{3,4}$ \\ 1 Departamento de Física, Universidad Católica del Norte, \\ Av. Angamos 0610, Antofagasta, Chile \\ ${ }^{2}$ Departamento de Física, \\ Facultad de Ciencias Exactas y Naturales, \\ Universidad Nacional de La Pampa, \\ CONICET, Av. Peru 151, 6300, \\ Santa Rosa, La Pampa, Argentina \\ ${ }^{3}$ Instituto de Física La Plata-CCT-CONICET, \\ Universidad Nacional de La Plata, \\ C.C. 727, 1900, La Plata, Argentina \\ 4 SThAR - EPFL, Lausanne, Switzerland
}

\begin{abstract}
We investigate the physics of a medium-size quantum particle' gases, of the order of hundreds or thousands of fermions/bosons (far below the thermodynamic limit $N \rightarrow 10^{24}$ ). Appeal to microcanonical ensemble becomes then unavoidable. The so-called statistical complexity is our protagonist. Interesting facts are uncovered, both for fermions and bosons, yielded by statistical quantifiers.

Keywords: Fermions, Bosons, Statistical complexity, Entropy, Disequilibrium.
\end{abstract}

\section{INTRODUCTION}

Examples of collective phenomena which can emerge from real-world complex systems include traffic congestion, financial market crashes, wars, and epidemics. They do not involve $10^{24}$ objects or agents but rather hundreds or thousands of them, so that canonical ensemble considerations may not apply. Instead, micro-canonical (MC) ones should be employed. Of course, they do not involve probabilities. We will appeal to MC techniques here, using the quantum micro-canonical formalism developed by Lemons. This approach is approximate, but a very good one [1].

The so-called statistical complexity $(C)$ is the protagonist of our story. It emerges when one attempts to quantify, for a given system, correlation structures. This involves the ability to discern the relations among a system's components by recourse to some specific complexity quantifier $C$. $C$ should be able to somehow mimic the manner in which entropy $S$ assess disorder. Two extreme scenarios might be thought of: (i) perfect order or (ii) maximal randomness, for which complexity should not be present [2]. In between, diverse correlation degrees may exist. One wishes that our putative quantity $C$ would be capable of quantifying them. How is one to represent $C$ ?

López-Ruiz, Mancini, and Calvet (LMC) [2] advanced, in what constituted a significant leap forward, an statistical complexity measure $C$ in the fashion

$$
C=D S,
$$

where $S$ is the entropy and $D$ a distance in probability space from the actual probability distribution to the uniform one. $D$ was baptized as the disequilibrium by LMC. If not null, this signals the presence of privileged states. Their $C$-proposal received both considerable attention and great interest [2-9]. It was applied in variegated scenarios for both the canonical and grand canonical ensembles. The corresponding $C$-micro-canonical version was advanced in Ref. [10]

We will here focus attention on a $C$-scenario for intermediate-size's sets of either fermions or bosons, using the Lemons quantum micro-canonical approach, approximate but very good [1]. We use quantifiers like $S, D$, and $C$ as defined in Ref. [10], that yield new useful information regarding the behavior of our free quantum particles' set. We will obtain revealing insights, notwithstanding the fact that we are dealing with free particles. The associated sets do exhibit complexity.

The paper is organized as follows. Section II us devoted to free fermion systems. As an application, we discuss some facets of semiconductors in Section III. We deal with free bosons in Section IV and draw some conclusions Section V. 


\section{A. Microcanonical disequilibrium and statistical complexity}

LMC defined $D$ as a distance in probability space: that to the uniform distribution. We work here in a scenario devoid of probabilities, a micro-canonical one. We proceed as in Ref. [10] and call $S_{\max }$ to the maximum possible entropy, using ideas of Ref. [11] (see also Ref. [3]). Thus, one writes for $D$

$$
D=1-\frac{S}{S_{\max }} .
$$

$D$ vanishes for $S=S_{\max }$ and is maximal for $S=0$, as one should expect. Accordingly, we have now a probability-less LMC complexity given by Eq. (1). We further define $H=S / S \max$ and finally write

$$
C=4 H(1-H)
$$

that is normalized in the sense of ranging between zero and unity.

\section{B. A motivating fact}

As a motivation consider the following graph for 100 bosons.

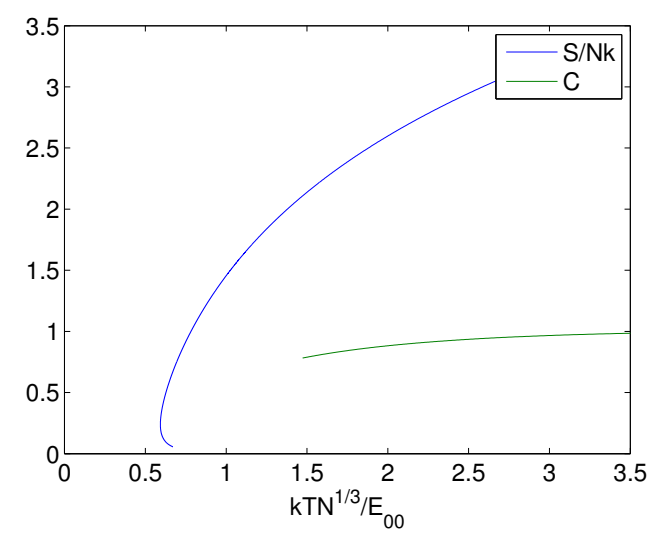

FIG. 1: Normalized entropy and statistical complexity versus temperature for an $N$-boson gas.

Our first graph here resembles Fig. 7.3 of Ref. [1] for the entropy $S$ but we add our quantifier $C$. The two quantities are plotted against a normalized temperature $T_{R}^{(B)}=k T N^{1 / 3} / E_{00}$ that will be defined below. Here just notice the kind of bizarre behavior of the two quantities at rather low temperatures. In particular, $C$ cannot be straightforwardly computed if $T_{R}^{(B)}$ is low enough. This cries for an explanation that we will attempt to provide below. In particular, we ask: what is $C$ telling us in this graph?

\section{N-FERMIONS SYSTEM}

\section{A. The microcanonical treatment}

The multiplicity $\Omega$ of a system of quantum particles composed of $N$ fermions that occupy volume $V$ and share an energy $E$ is given by [1]

$$
\Omega(E, V, N)=\left[V\left(\frac{E}{N}\right)^{3 / 2}\left(\frac{4 \pi e m}{3 h^{2}}\right)^{3 / 2}\right]^{N}
$$


If $n$ is the number of fermion microstates available to $N$ fermions, each with energy $E / N$, it is shown in Ref. [1] that

$$
n=\Omega(E / N, V, 1)=V\left(\frac{E}{N}\right)^{3 / 2}\left(\frac{4 \pi e m}{3 h^{2}}\right)^{3 / 2} .
$$

Here $e$ is the basis of natural logarithms, $m$ the fermion mass, and $h$ Planck's constant. Because at most one fermion may occupy the same microstate one has

$$
0<N / n \leq 1
$$

If every one of the available single particle microstates is filled with one fermion, that is when $N=n$ and the volume remains at $V$, the associated energy $E_{0}$ becomes the ground state energy, i.e., it is the lowest possible internal energy (Fermi level). Thus, from above equation we have [1]

$$
E_{0}=\frac{N^{5 / 3}}{V^{2 / 3}}\left(\frac{3 h^{2}}{4 \pi e m}\right)
$$

and then

$$
\frac{E}{E_{0}}=\left(\frac{n}{N}\right)^{2 / 3}
$$

For $V$ a cube of 50 Angstrom' sides and 100 electrons one has $E_{0} \sim 8.8 \mathrm{eVs}$. We will employ below normalized fermion temperatures defined as

$$
T_{R}^{(F)}=\frac{N k T}{E_{0}}
$$

(Here we energetically re-normalize the temperature scale and work with re-normalized temperatures $T_{R}^{(F)}$ ). Returning to the multiplicity, we have in general

$$
\Omega(n, N)=\frac{n !}{(n-N) ! N !},
$$

distinct ways of filling $n$ microstates with $N$ fermions. Accordingly, following the celebrated equation $S(n, N)=$ $\ln \Omega(n, N)$ engraved in L. Boltzmann's tomb at Vienna's cemetery, the entropy obtained from Eq. (10) is

$$
S=k \ln \left(\frac{n !}{(n-N) ! N !}\right) .
$$

When the average occupancy $N / n$ is low, that is $N / n \ll 1$ the entropy becomes the Sackur-Tetrode classical entropy for an system of free particles [1], which is given by

$$
S_{\text {class }} / N k=\frac{5}{2}+\ln \left[\frac{V}{N}\left(\frac{E}{N}\right)^{3 / 2}\left(\frac{4 \pi m^{3 / 2}}{3 h^{2}}\right)\right] .
$$

Introducing (7) into the above equation we are led to

$$
S_{\text {class }} / N k=1+\ln \left(\frac{n}{N}\right)
$$

as shown by Lemons in his book [1]. We display the behavior of i) the entropies $S$, ii) $T$, and iii) the specific heat $C_{V}$, as a function of $N / n$ (running from 0 to 1 ), in Fig. 2.

We see that $C_{V}$ tends to its classical $3 / 2$ value at very low $N / n$. The three quantities $S, T$, and $C_{V}$ vanish at the ground state, respecting thermodynamics third law. Fig. 3 depicts $S$ and $C$ versus $N / n$ for the same range of $N / n$. As expected, the complexity vanishes at the classical limit $(N / n \ll 1)$ and at the ground state. It reaches a maximum at an $N / n$ value that signals the beginning of the classical $N / n$ range of values. In Fig. 4 we depict $C$ in an ample $T$ - range to show that it tends to vanish for very high temperatures. 


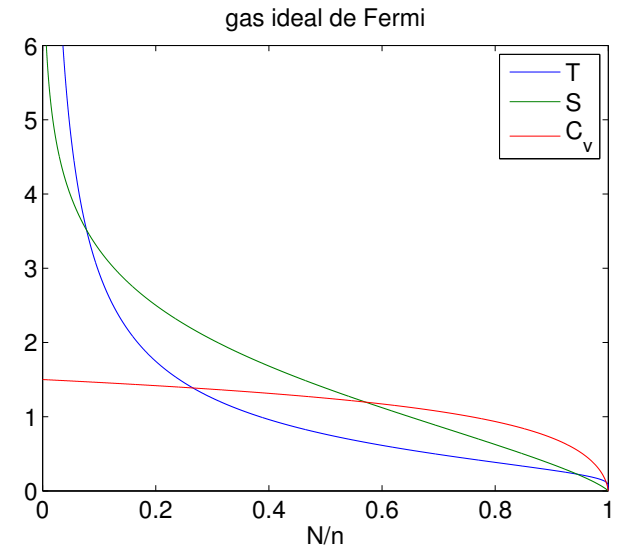

FIG. 2: $T, S$, and specific heat $C_{V}$, as a function of $N / n$ (running from 0 to 1 ).

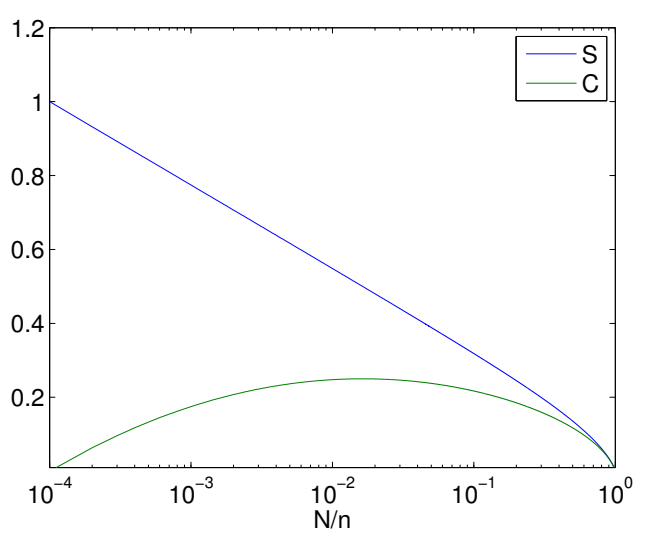

FIG. 3: $S$ and $C$ versus $N / n$.

\section{B. Equations of state}

Since the entropy (11) and the occupancy $N / n$ completely describe the thermodynamics of an system fermions, the appropriate equations of state are easily derived from them [1]

$$
\frac{E}{N k T}=-\frac{3}{2} \frac{n}{N} \ln (1-N / n)
$$

and

$$
\frac{P V}{N k T}=-\frac{n}{N} \ln (1-N / n)
$$

where the temperature is defined via $1 / T=(\partial S / \partial E)_{V, N}$ and the pressure according to $P / T=(\partial S / \partial V)_{E, N}$. From Eqs. (7) and (14) we find a temperature

$$
T=-\frac{2}{3} \frac{E_{0}(N / n)^{1 / 3}}{N k \ln (1-N / n)} .
$$

At this stage we can also obtain the specific heat as $C_{V}=T(\partial S / \partial T)_{V}$. In Fig. 5 we show both $S / S_{\max }$ and $C$ this time versus the normalized temperature $T_{R}^{(F)}$.

The complexity maximum appears at the $T_{R}$-value high enough to reach the classical regime. Against the normalized temperature we plot also $N / n$ and $C_{V}$ in Fig. 6 . 


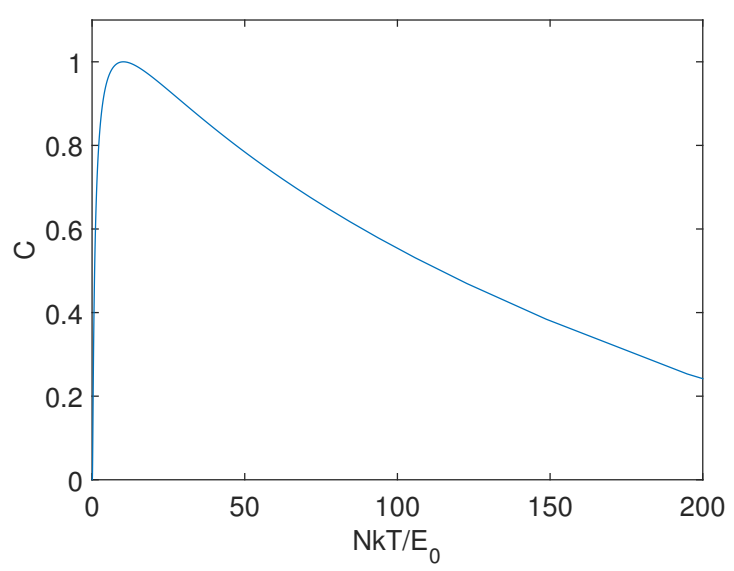

FIG. 4: $C$ versus $T_{R}^{(F)}=N k T / E_{0}$. $C$ vanishes as $T$ grows without bound.

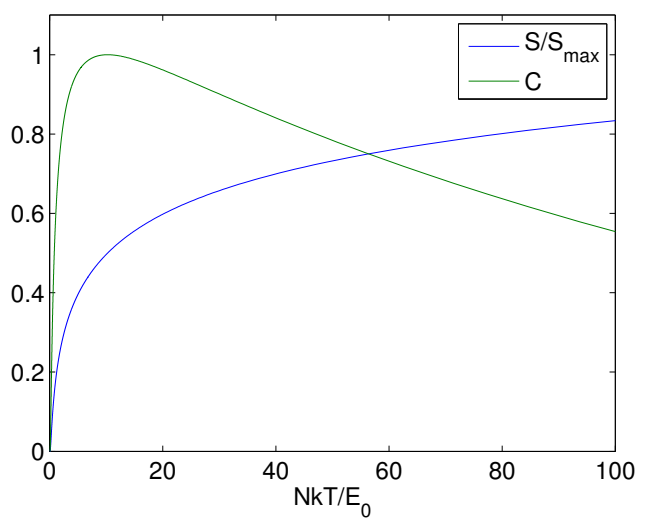

FIG. 5: $S / S_{\max }$ and $C$ versus the normalized temperature $T_{R}^{(F)}$.

Clearly, at high temperatures the specific heat reaches its classical value of 1.5 . The two quantities $C$ and $C_{V}$ are now plotted against $N / n$ in Fig. 7. Compare with Fig. 3 for $C$. The maximum complexity is attained as we reach the classical limit.

Here, in the two limits (high and low $N / n$ ), associated with, respectively, the classical limit and the zero- $T$ one, $C_{V}$ behaves as expected. It vanishes at zero $T$ and attains its classical value $3 / 2$ for high temperatures. This is confirmed in Fig. 6, described above, where the specific heat is plotted against the normalized $T$. Finally, we plot $C$ versus $C_{V}$ in Fig. 8.

We see here that the complexity vanishes at the classical limit, when $C_{V}$ attains its classical value. This figure also shows that $C$ prefigures (or announces) that we are reaching the classical limit by attaining a maximum shortly before this happens. This $C$-property has been observed in other scenarios [9].

Let us consider Gibbs' free energy $G$. We have

$$
G=E-T S+P V
$$

with $E, S, P V$, and $T$ given by, Eqs. (8), (11), (15), and (16), respectively. From Eqs. (15) and (16) we find

$$
P V=\frac{2}{3} E_{o}\left(\frac{N}{n}\right)^{-2 / 3}
$$

that together with Eq. (8) yields 


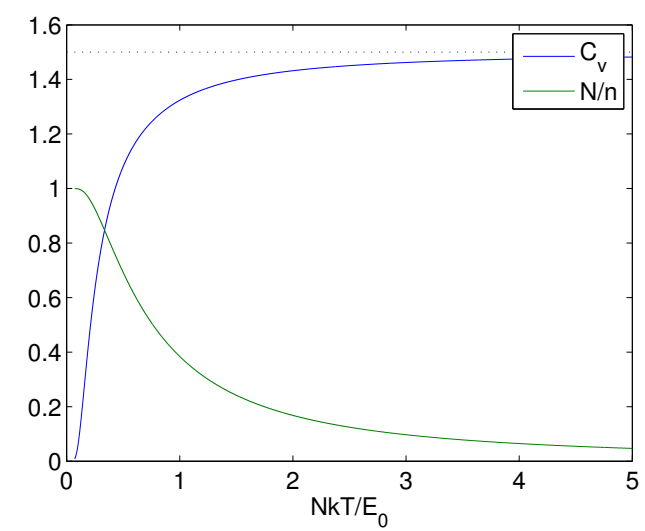

FIG. 6: $C_{V}$ and $N / n$ versus $T_{R}^{(F)}$.

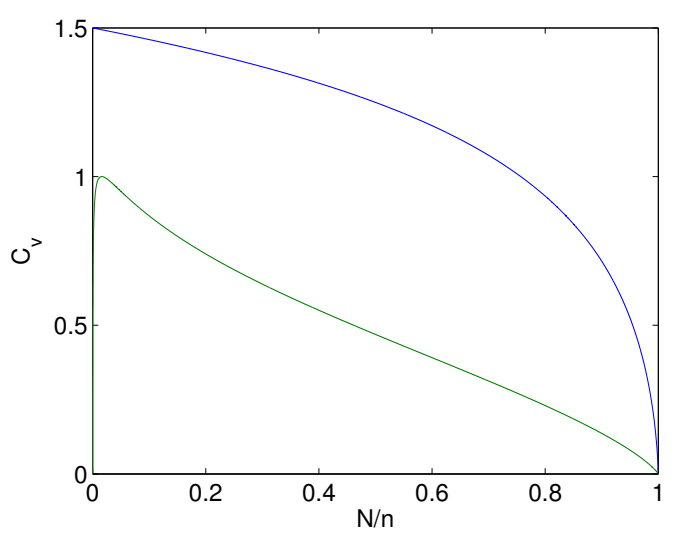

FIG. 7: $C$ (green) and $C_{V}$ (blue) versus $N / n$.

$$
G=\frac{5}{3} E_{o}\left(\frac{N}{n}\right)^{-2 / 3}-T S
$$

We plot $G$ in Figs. 9 and 10 in terms of respectively, $T_{R}^{(F)}$ and $N / n$. We see that $G$ signals the classical limit as well, by becoming negative when the horizontal coordinate reaches the classical zone, which is absurd since we deal with free particles.

Summing up, a finite $N$-set of free fermions does display structural details that are revealed by statistical quantifiers.

\section{A SIMPLE MODEL FOR SEMICONDUCTORS AS A FERMION-APPLICATION}

We consider that an intrinsic semiconductor (IS) is a crystal with no defects or impurities [1]. If lattice vibrations excite a valence band (BV) electron so that it reaches the IS-conduction band (CB), both the hole left in the BV and the new arrival CB-electron are free to move if affected by an applied electric field. Holes and electrons equally contribute to the IS-conductivity. In a simplified model [1], the numbers of single-particle microstates (SPME) for BV and CV are equal. Same for the number of holes and conduction electrons. The valence and conducting bands of a more complete model would consist of closely spaced non-degenerate energy levels. We consider here a simple model [1] that captures one important IS-feature, the band-gap energy $\epsilon$, while ignoring other features. The VB-arrangement of holes and that of electrons in the CB both contribute to the multiplicity of an intrinsic semiconductor. Assume that the semiconductor-model has $N$ electrons, and, as regards micro-states, $N$ VB-SPME, and $N$ CV-SPME. A word of caution. Now $n$ and $N$ do not have the same meaning that they do in the preceding Section. 


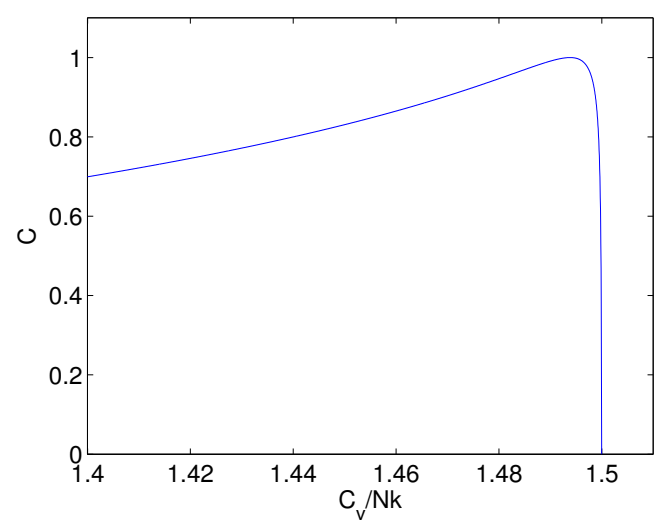

FIG. 8: $C$ versus $C_{V} / N k$.

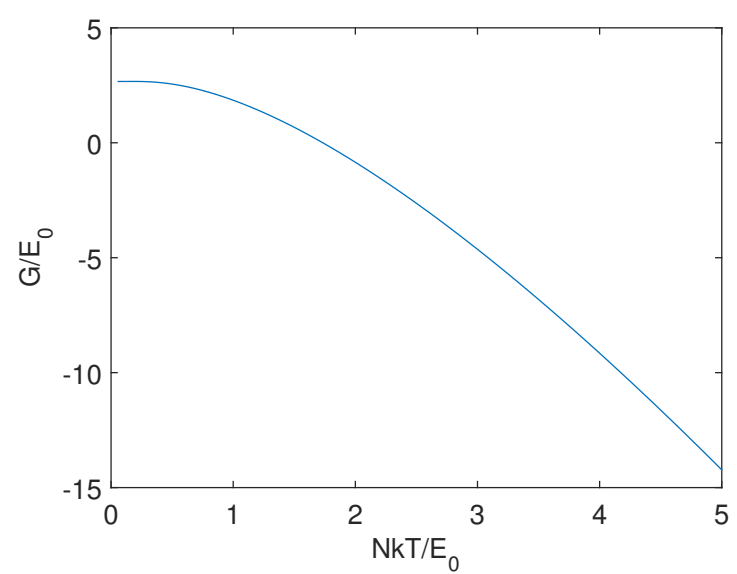

FIG. 9: $G$ versus temperature $T_{R}^{(F)}$.

Out of these $N$ electrons, $n$ occupy the CB. Typically, $n$ is of the order of $10^{7}[1]$. Since the number of electrons is conserved, $N$ minus $n$ are left to occupy the valence band. Thus, $N ! /(n !(N-n) !)$ is the number of equally probable VB-configurations of the VB-subsystem. The reasoning line gives an identical results for the CB arrangements. Accordingly, the multiplicity $\Omega$ of our IS-model is [1]

$$
\Omega=\left(\frac{N !}{(n !(N-n) !}\right)^{2},
$$

with an associate entropy $S$ and energy $E[1]$

$$
S=k \ln \Omega,
$$

$$
E=n \epsilon .
$$

Roughly, $E$ is of the order of $10^{7} \mathrm{eV}[1]$. Because we assume that there are no inter-particle forces, we are allowed to speak of an ideal Fermi gas. 


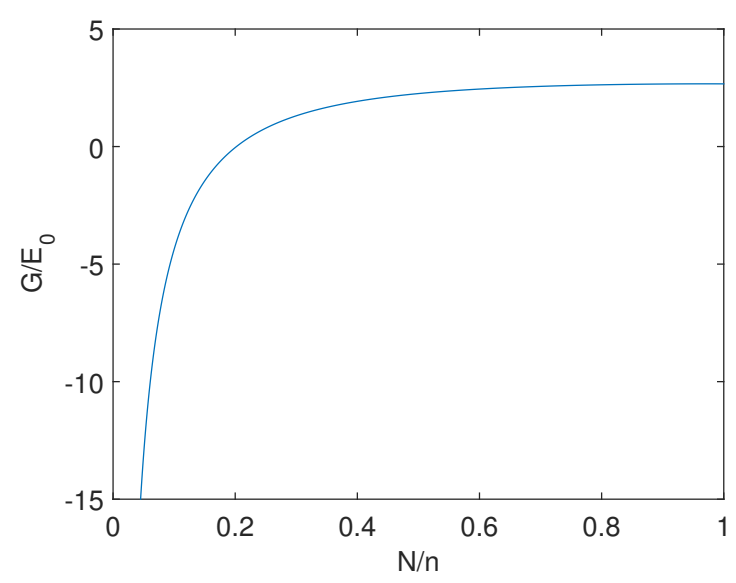

FIG. 10: $G$ versus $N / n$.

\section{A. Entropy}

Our Fermi entropy can be written in the fashion (for details see Ref. [1])

$$
S=2 k[N \ln N-n \ln n-(N-n) \ln (N-n)]
$$

and one can recast this as [1]

$$
S=-2 N k[(E / N \epsilon) \ln (E / N \epsilon)-(1-E / N \epsilon) \ln (1-E / N \epsilon),]
$$

and call

$$
x=E / N \epsilon,
$$

with

$$
0 \leq x \leq 1
$$

We assume always [1] $N \gg n$ and $n \gg 1$. The temperature of the system is obtained from the thermodynamical entropy. Thus, in this case, we have [1]

$$
\frac{1}{T}=\frac{\partial S}{\partial E}=(2 / \epsilon) \ln \left(N \epsilon E^{-1}-1\right)
$$

Thus, one has $[1]$

$$
\beta=(2 / \epsilon) \ln \left(N \epsilon E^{-1}-1\right),
$$

or

$$
\beta=(2 / \epsilon) \ln (1 / x-1) .
$$

The equation of state ensues [1]

$$
x=\frac{1}{\exp (\beta \epsilon / 2)+1} .
$$




$$
E=\frac{N \epsilon}{1+\exp (\beta \epsilon / 2)} .
$$

This equation, together with $E=n \epsilon$ immediately yields [1]

$$
\frac{n}{N}=\frac{1}{1+\exp (\beta \epsilon / 2)}
$$

In a typical semiconductor we find band gap energies of the order of a few electron-volts $\left(1 \mathrm{eV}=1.60 \times 10^{-19}\right.$ joules [1]). For $\epsilon=2 \mathrm{eV}$, the frequency of occupied conduction band single-particle micro-states $n / N$ at room temperature $\left(T=300^{\circ} \mathrm{K}\right.$ and $\left.k T=0.026 \mathrm{eV}\right)$, is of the order of $2 \times 10^{-17}$, a very small number that does contribute to the IS-conductivity [1]. We introduce now a (different from that for fermions of above) semiconductor-normalized temperature $T_{R}^{(S)}=k T / \epsilon$ and plot $S / S_{\max }, n / N$, and $C$ versus $T_{R}^{(S)}$ in Fig. 11. We see that $S$ starts from zero and null temperature and steadily grows till saturation.

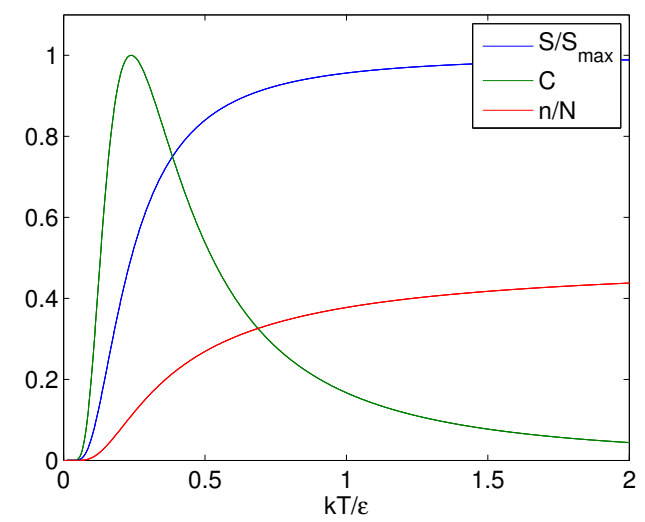

FIG. 11: $S / S \max , C$, and $n / N$ versus $T_{R}^{(S)}$.

Note the pronounced maximum displayed by the complexity. In order to understand it look now at Fig. 12, that displays $C$ together with $\mathrm{d} S / \mathrm{d} T$. We see that $C$ is maximal at that temperature at which the growth of $S$ towards saturation is speediest.

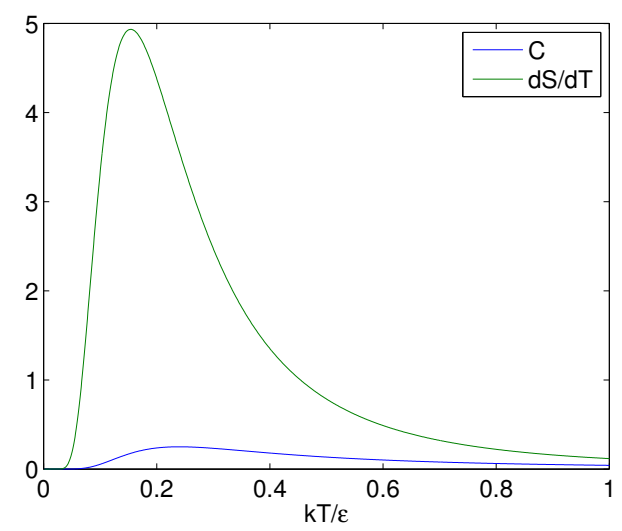

FIG. 12: $C$ together with $\mathrm{d} S / \mathrm{d} T$ versus $T_{R}^{(S)}$. Their respective maxima almost coincide.

The two maxima almost coincide. As before, the $C$-maximum slightly precedes the other maximum. Fig. 11 is also relevant, displaying $S$ and $C$ versus $T_{R}^{(S)}$. Next, consider Fig. 13. It depicts $n / N$ and its derivative with respect to 
$T_{R}^{(S)}$ versus the re-normalized temperature. We now realize that $C$ is maximal at that $T_{R}^{(S)}$ for which $\partial(n / N) / \partial T_{R}^{(S)}$ is maximal too.

At $T=0$ all $N$ electrons remain in the valence band. As $T$ grows, $n$ electrons jump to the conduction band. $n$ augments as $T$ increases. $n / N$ is the electron-fraction that has been removed from the VB.

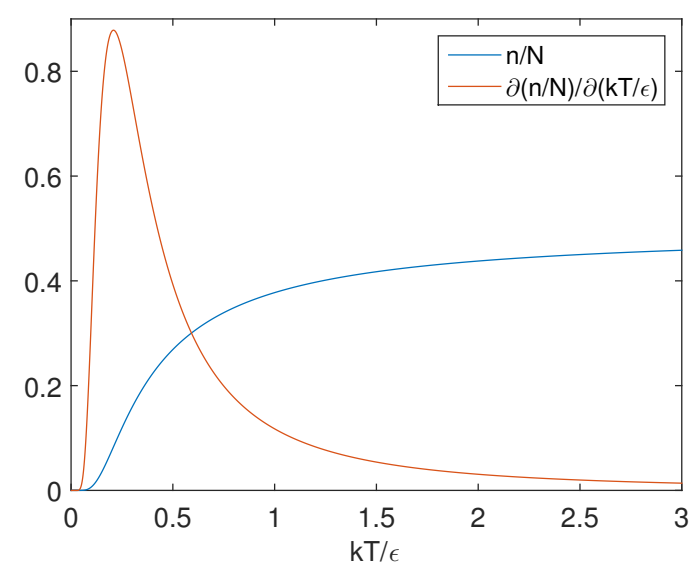

FIG. 13: $n / N$ and $\mathrm{d}(n / N) / \mathrm{d} T_{R}^{(S)}$ versus $T_{R}^{(S)}$.

We conclude that the $C$-maximum signals the maximum rate of electron-removal from the valence band.

\section{N-BOSONS SYSTEM}

Here $n$ stands, as in Section 2, for the number of accessible single-particle micro-states, given by [1]

$$
n=\Omega(E / N, V, 1)=V\left(\frac{E}{N}\right)^{3 / 2}\left(\frac{4 \pi e m}{3 h^{2}}\right)^{3 / 2} .
$$

Because any number of bosons may occupy the same micro-state one has

$$
0<N / n \equiv x \leq N
$$

The multiplicity, i.e., the number of distinct ways of placing $N$ identical bosons, each with energy $E$ in $n$ different micro-states is (consider $n-1$ partitions separating groups of bosons) [1]

$$
\Omega=\frac{(N+n-1) !}{N !(n-1) !}
$$

so that

$$
S=k \ln \Omega(E, V, N) \text {. }
$$

Except for the ground state (see below) we may apply Stirling's approximation when dealing with hundreds of bosons. Then, we have that [1]

$$
S / N k=x^{-1} \ln (1+x)+\ln \left(1+x^{-1}\right) .
$$

The classical limit is obtained for $x \ll 1[1]$. The equations of state are [1]

$$
\frac{E}{N k T}=\frac{3}{2} x^{-1} \ln (1+x)
$$




$$
\begin{gathered}
\frac{P V}{N k T}=x^{-1} \ln (1+x), \\
P V=\frac{3}{2} k T .
\end{gathered}
$$

The real ground state of an ideal Bose system is the one for which the $N$ bosons occupy the same micro-state. Thus, $n=1$, and will call the energy of this special ground state $E_{00}$, which from (33) is obtained setting there $n=1$, i.e.,

$$
1=V\left(\frac{E_{00}}{N}\right)^{3 / 2}\left(\frac{4 \pi e m}{3 h^{2}}\right)^{3 / 2}
$$

so that

$$
E_{00}=\frac{N}{V^{2 / 3}} \frac{3 h^{2}}{4 \pi e m}
$$

The numerical value of $E_{00}$ for $N=100$ and $V$ a cube of side 50-Angstroms, with the mass of a Helium-4 atom, is $1.45 \times 10^{-} 4-\mathrm{eV}$.

Note that in the ground state's case Stirling's approximation cannot be employed. Remark that here the classical limit is reached for $n / N \gg 1$. The normalized boson temperature now becomes [1]

$$
T_{R}^{(B)}=\frac{k T N^{1 / 3}}{E_{o o}}=\frac{2}{3} \frac{x^{1 / 3}}{\ln (1+x)} .
$$

This re-normalized temperature has a rather short "intermediate" range between the ground state (GS) and the classical limit, as seen in Fig. 14.

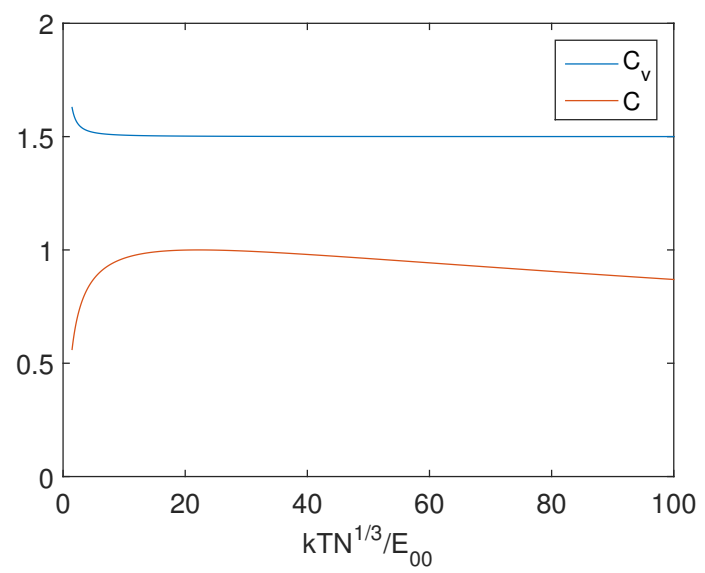

FIG. 14: $C_{V}$ and $C$ versus temperature $T_{R}^{(B)}$.

Note that $C_{V}$ is zero for the ground state and $3 / 2$ at the classical limit. $T_{R}^{(B)}=1.62$ at the GS and equals 21.62 for $C_{V}$ very near $3 / 2$. This entails a "useful" $T_{R}^{(B)}$-range of about 20 units.

\section{A. Bosonic results}

Fig. 15 depicts, versus $n / N, S / N k, C_{V} / N k$, and $C$, respectively. 


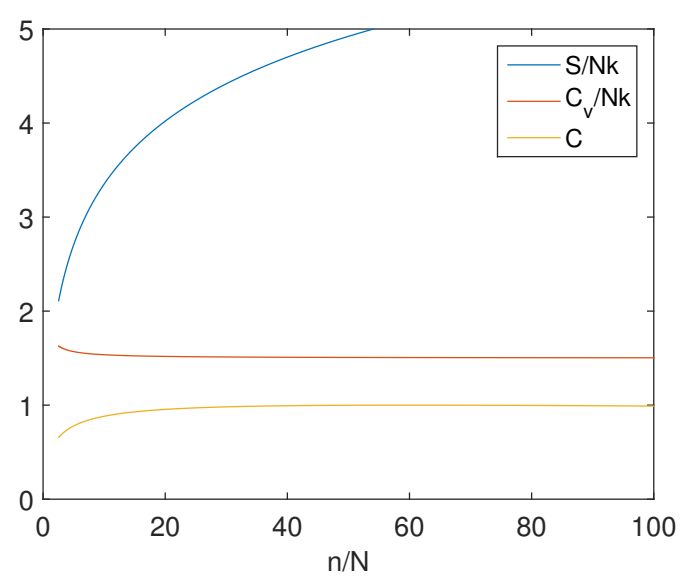

FIG. 15: $S / N k, C_{V} / N k$, and $C$ versus $n / N$.

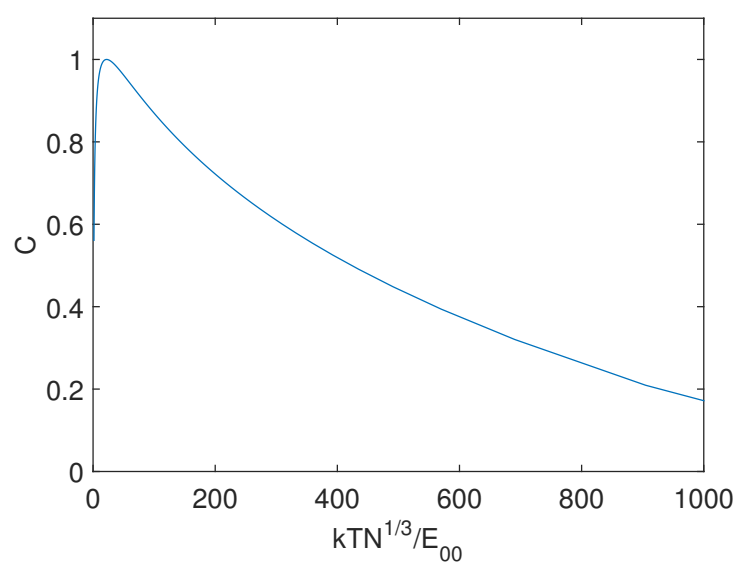

FIG. 16: $C$ vs. temperature $T_{R}^{(B)}$. $C$ vanishes as $T \rightarrow \infty$.

The classical limits lies at the extreme right, where $C_{V}$ tends towards its classical value. In Fig. 16 we appreciate that $C$ has a maximum in approaching the classical limit and then tends to zero when $T$ grows without bounds.

At the extreme left we detect a sort of cut-off. More precisely, some technical difficulties arise in he drawing program near the vertical axis. The same effect is observed is we plot $C$ and $C_{V}$ vs. $T_{R}^{(B)}$, as in Fig. 17. Why?

Because, as shown in Ref. [1], there is a critical $N / n=0.392$-value and a critical low temperature $T_{R}^{(B)}=T_{c}=1.48$ at which the Bose-condensate emerges. Two phases enter the scene here. The "gas" phase exists from $N / n_{c}=0.392$ down to $N / n=0$. In $T$ terms this is equivalent to the range $\left[T_{c}=1.48-\infty\right]$. Of course, for $n / N$ we have the range $[2.551-\infty][1]$

From $T_{c}$ downwards, or from $N / n=0.392$ upwards [1], the Bose-condensate arises. The operating equations here are (38) and (43). They allow one to compute $S$ in terms of $x$ and also in terms of $T$. One can then calculate all quantities in the "gas" phase for $x>0.392$. In the condensate we have $N_{0}$ particles and the remaining ones, $N_{u}$ (with x=0.392), belong to the "gas" phase. Of course, $N=N_{0}+N_{u}$.

Remark that $x$ does not vary continuously in the $x$ - range $[0.392,0]$. For $T=0$ we have $x=N$ and $S=T=C_{V}=0$.

See Fig. 18 that displays $x$ vs. temperature, with the discontinuity at $T_{R}^{(B)}=T_{c}=1.48[1]$.

In the $T$-range $\left[T_{c}, 0\right]$, we have $[1]$

$$
\frac{S}{N k}=2.11\left(\frac{T}{T_{c}}\right)
$$




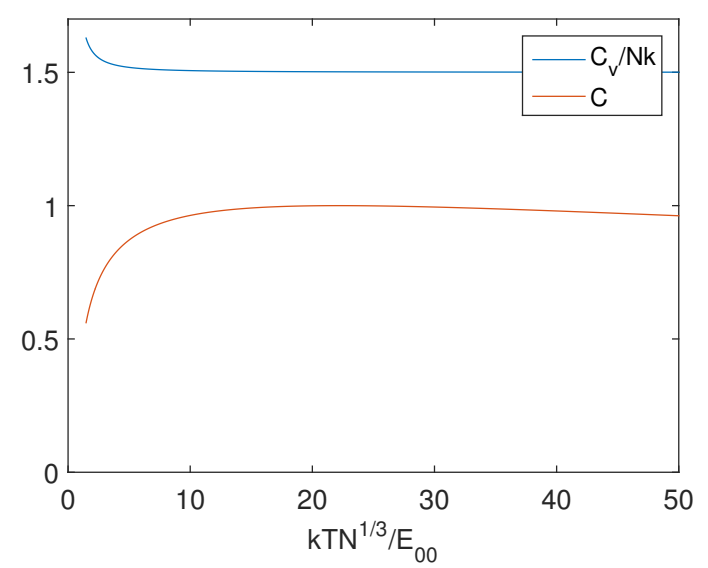

FIG. 17: $C$ and $C_{V}$ vs. $T_{R}^{(B)}$.

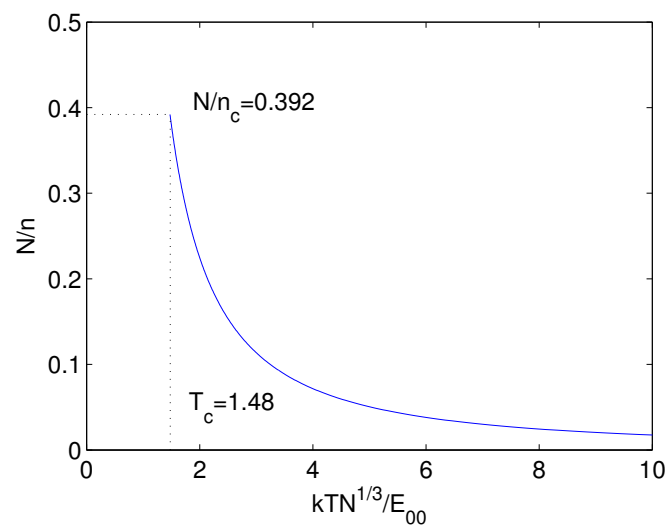

FIG. 18: $N / n$ vs. temperature, with a discontinuity at $T_{R}^{(B)}=T_{c}=1.48$.

from which we can compute, for the condensate, $C$ and $C_{V}$. One sees that $C_{V}=T \mathrm{~d} S / \mathrm{d} T$ displays a discontinuity at $T_{c}$ as discussed below. In Fig. 19 we plot three quantities. They are $S, C_{V}$, and $C$, against $T_{R}^{(B)}$.

The phase transition gas-condensate is clearly appreciated for 100 bosons, as illustrated in the already mentioned Fig. 19. One clearly appreciates the distinctive behavior of $S, C_{V}$, and $C$ at the phase-transition. Fig. 18 displays $x$ vs. temperature, with the discontinuity at $T_{c}$. A different, instructive view of the phase transition is offered by Fig. 20, that gives $C$ vs. $C_{V}$. The phase transition gas-condensate is clearly appreciated for 100 bosons. 


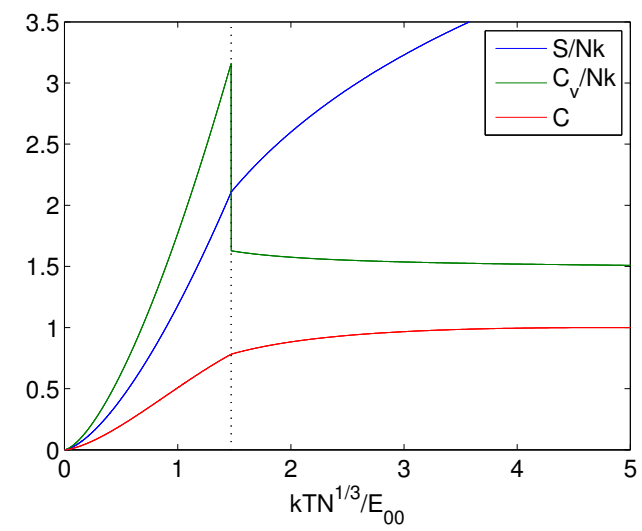

FIG. 19: $S / N k, C_{V} / N k$, and $C$, against $T_{R}^{(B)}$.

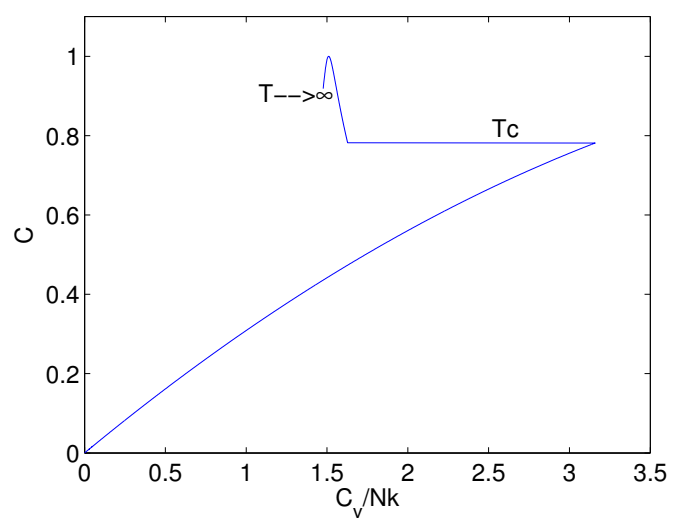

FIG. 20: $C$ vs. $C_{V} / N k$.

\section{CONCLUSIONS}

We have seen that a system of $N$ free fermions (bosons) displays several interesting physical features, notwithstanding its apparent simplicity. We have shown that these features are adequately described by statistical quantifiers. Amongst them, the statistical complexity is always able to detect two kinds of transitions

- from the quantal to the classical regimes,

- for bosons, from the gas phase to the condensate one.

It is rather impressive also that $C$, in semiconductors, becomes maximal at that temperature at which the rate of electron-removal from the valence band is a maximum as well.

\section{Acknowledgment}

Research was partially supported by FONDECYT, grant 1181558 and by Conicet (Argentine Agency).

\section{Author contribution}

All authors contributed equally to the manuscript, read and approved the final manuscript. 


\section{Conflict of interests}

The authors declare no conflict of interest.

[1] Lemons, D.S. A Student's Guide to Entropy. Cambridge University Press, 2014.

[2] López-Ruiz, R.; Mancini, H.L.; Calbet, X.; A statistical measure of complexity. Phys. Lett. A 1995, 209, 321-326.

[3] Martin, M.T.; Plastino, A.; Rosso, O.A. Statistical complexity and disequilibrium. Phys. Lett. A 2003, 311, $126-132$.

[4] Rudnicki, L.; Toranzo, I.V.; Sánchez-Moreno, P.; Dehesa, J.S. Monotone measures of statistical complexity. Phys. Lett. A 2016, 380, 377-380.

[5] López-Ruiz, R.; Mancini, H.; Calbet, X. A Statistical Measure of Complexity in Concepts and recent advances in generalized information measures and statistics, A. Kowalski, R. Rossignoli, E. M. C. Curado (Eds.), Bentham Science Books, pp. 147-168, New York, 2013.

[6] Sen, K.D. (Editor). Statistical Complexity, Applications in electronic structure, Springer, Berlin, 2011.

[7] Mitchell, M. Complexity: A guided tour, Oxford University Press, Oxford, England, 2009.

[8] Martin, M.T.; Plastino, A.; Rosso,O.A. Generalized statistical complexity measures: geometrical and analytical properties. Physica A 2006, 369, 439-462.

[9] Pennnini, F.; Plastino, A. Disequilibrium, thermodynamic relations, and Rényi's entropy. Phys. Lett. A 2017, 381, $212-215$.

[10] Pennini, F.; Plastino, A. Statistical complexity without explicit reference to underlying probabilities. Physica A 2018, 500, 121-130.

[11] Shiner, J.S.; Davison, M.; Landsberg, P.T. Simple measure for complexity. Phys. Rev. E 1999, 59, 1459-1464. 